\title{
Meaningful Scales Detection: an Unsupervised Noise Detection Algorithm for Digital Contours
}

\author{
Bertrand Kerautret $^{1}$, Jacques-Olivier Lachaud ${ }^{2}$ \\ ${ }^{1}$ LORIA, Université de Lorraine (France) (bertrand.kerautret@loria.fr) \\ ${ }^{2}$ LAMA, Université de Savoie (France) (jacques-olivier.lachaud@univ-savoie.fr) \\ Communicated by Bertrand Kerautret Demo edited by Bertrand Kerautret
}

\begin{abstract}
This work presents an algorithm which permits to detect locally on digital contours what is the amount of noise estimated from a given maximal scale. The method is based on the asymptotic properties of the length of the maximal segment primitive.
\end{abstract}

\section{Source Code}

The implementation of the algorithm is available through the ImaGene ${ }^{1}$ library framework. A special version of this library is given without boost and gmp dependencies. The source code and the online demonstration are accessible at the IPOL web page of this article ${ }^{2}$.

Keywords: noise estimation; meaningful scale detection

\section{Overview}

The estimation of the meaningful scale of a digital contour is a difficult problem that can have important impacts for numerous applications which are dependent of a supervised noise parameter. For instance, it is the case in various curvature estimators $[4,9,10]$ or in contour polygonalisation algorithms $[1,11]$.

This work presents an algorithm which permits to detect locally what is the amount of noise estimated from a given maximal scale. The method is based on the asymptotic properties of the length of the maximal segment primitive [7]. The presented algorithm is related to previous work which was first presented in conference [5] and then further extended [6].

\footnotetext{
${ }^{1}$ ImaGene, \{Gen\}eric Digital \{Ima\}ge Library, http://gforge.liris.cnrs.fr/projects/imagene

${ }^{2}$ https://doi.org/10.5201/ipol.2014.75
} 


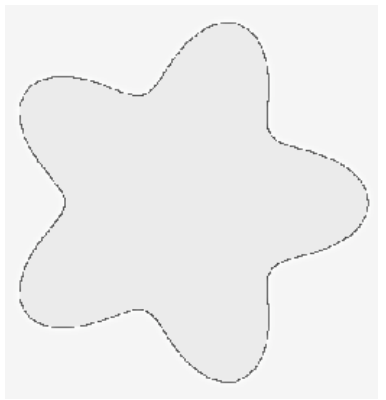

$\mathrm{X}$

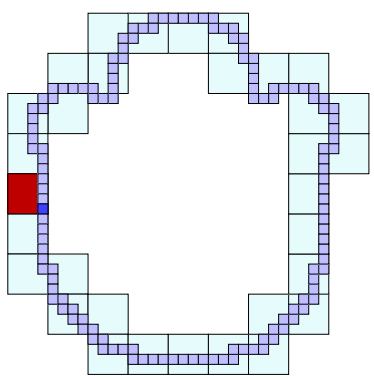

$\left(x_{0}, y_{0}\right)=(0,0)$

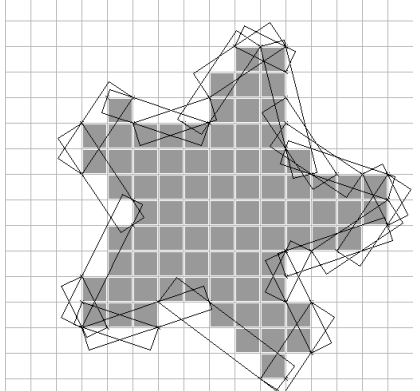

$\operatorname{Dig}_{2}(X)$

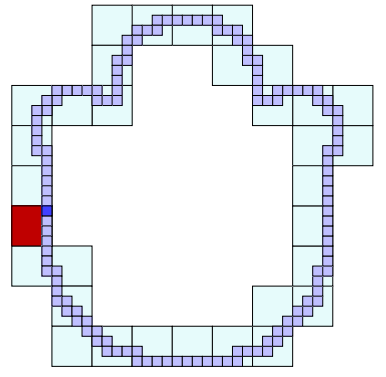

$\left(x_{0}, y_{0}\right)=(0,3)$

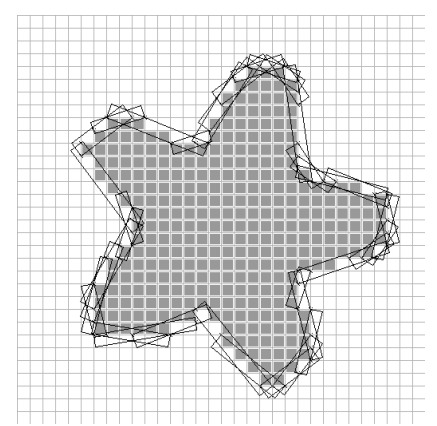

$\operatorname{Dig}_{1}(X)$

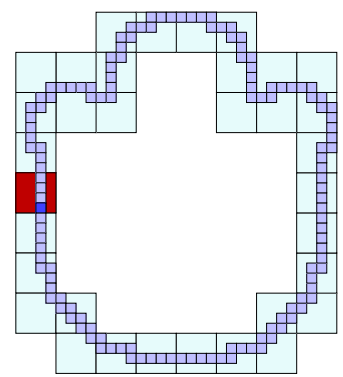

$\left(x_{0}, y_{0}\right)=(1,0)$

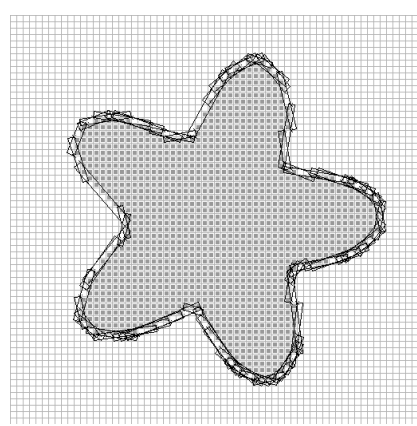

$\operatorname{Dig}_{0.5}(X)$

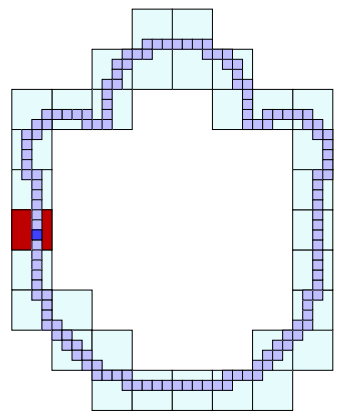

$\left(x_{0}, y_{0}\right)=(1,1)$

Figure 1: First row: Illustration of the digitization process $\operatorname{Dig}_{h}()$ applied on the real shape $X$ with the set of maximal segments covering the digital contours. Second row: Illustration of several subsampling with the same digitization grid size but with different shifts $\left(x_{0}, y_{0}\right)$.

\section{Method}

The method is based on the study of the properties of the maximal digital straight segment which is a classic primitive used in the field of discrete geometry. This primitive is for instance used for tangent or curvature estimators. As mentioned in the introduction, the length of the maximal segments is exploited to detect a meaningful scale through a given maximal scale. An illustration representing this primitive with finer and finer scale is given in figure 1 (first row) where maximal segments are represented by the boxes. Before describing the asymptotic property on the length of this primitive, we briefly recall its classic definition.

\subsection{Primitive of the Maximal Digital Straight Segment}

Definition of a Standard Digital Straight Line (DSL) A Standard Digital Straight Line $(D S L)$ is some set $\left\{(x, y) \in \mathbb{Z}^{2}, \mu \leq a x-b y<\mu+|a|+|b|\right\}$, where $(a, b, \mu)$ are also integers and $\operatorname{gcd}(a, b)=1$. The real lines of equation $a x-b y=\mu$ and $a x-b y=\mu+|a|+|b|-1$ are respectively the lower and upper leaning lines (as illustrated in figure 2 (a)).

Definition of a Maximal Straight Segment A Digital Straight Segment (DSS) is a 4-connected piece of DSL. The interpixel contour of a simple digital shape is a 4-connected closed path without self-intersections. A maximal segment $M$ of a 4-connected path $C$, is a subset of $C$ that is a DSS and which is no more a DSS when adding any other point of $C \backslash M$.

The figure figure 2 (b) illustrates the recognition process of a maximal segment starting from the point A. The points P1, Q1, P2, Q2, P3, Q3, P4, Q4, P5, Q5, Q6, Q7, Q8, and Q9 are added alternately to the front and to the back of the current segment. 


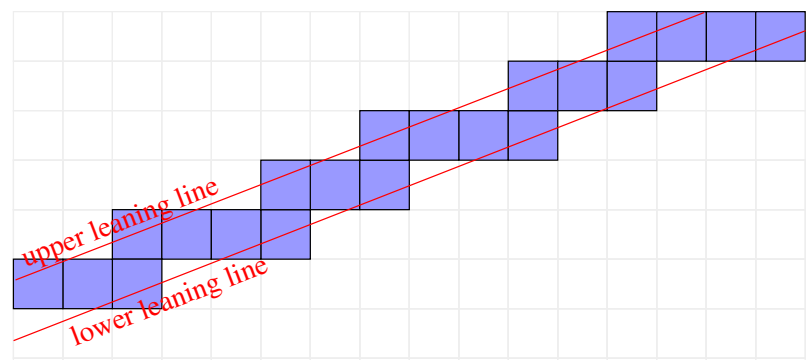

(a)

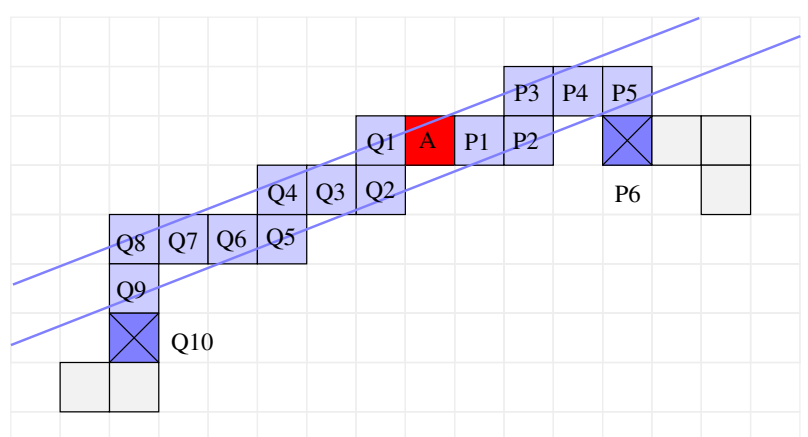

(b)

Figure 2: Illustration of a Digital Straight Line (a) and example of a maximal segment recognition process (b).

Extraction of Maximal Straight Segments through the ImaGene Library The maximal segment recognition algorithm is included in the ImaGene ${ }^{3}$ library. The class C4CTangentialCover ${ }^{4}$ is defined to compute all the set of maximal segments (class MaximalSegment ${ }^{5}$ ) associated to a digital contour (a 4-connected contour represented by the class C4CIterator ${ }^{6}$ ).

\subsection{Asymptotic Properties of Maximal Straight Segments}

The previous primitive presents some particular asymptotic properties with its length in number of steps (or number of pixel minus one). The asymptotic behavior is defined by considering different digitization steps $h$ of a real shape $X$.

More precisely, theorem 5.26 of a research report by Lachaud [7] (in chapter 5) indicates that given a real shape $X$, if we consider a digitization process $\operatorname{Dig}_{h}(X)=X \cap h \mathbb{Z} \times h \mathbb{Z}$ and if the shape $X$ has a $C^{3}$ boundary and is strictly convex, then the smallest discrete length of the maximal segment of $\operatorname{Dig}_{h}(X)$ is some $\Omega\left(1 / h^{1 / 3}\right)$. The longest discrete length of the maximal segments on the boundary of $\operatorname{Dig}_{h}(X)$ is some $O\left(1 / h^{1 / 2}\right)$. The experiments of figure 3 (a) confirm such a behavior.

However, since the shapes obtained with smaller and smaller digitization grid sizes are in general not available, we choose another strategy by subsampling the initial shape to obtain coarser and coarser shapes. The second line of figure 1 shows an example of several subsamplings defined with various shifts $\left(x_{0}, y_{0}\right)$. The lengths are thus defined for a given subsampling (characterized by the size $h_{i}$ and shift $\left.x_{0}, y_{0}\right)$ by the average. If we denote by $\left(L^{h_{i}, x_{0}, y_{0}}\right)_{j=1 . . l_{i}}$ the discrete lengths of the set of $l_{i}$ maximal segments covering $P$, the average length $\bar{L}^{h_{i}}$ is defined by:

$$
\bar{L}^{h_{i}}=\frac{1}{i^{2}} \sum_{0 \leq x_{0}, y_{0}<i} \frac{1}{l_{i}^{x_{0}, y_{0}}} \sum_{j} L_{j}^{h_{i}, x_{0}, y_{0}}
$$

where $l_{i}^{x_{0}, y_{0}}$ represents the number of maximal segments containing the subsampled point $P$.

By using this strategy we can observe a similar behavior as shown on the experiments of figure 3 (b).

\footnotetext{
${ }^{3}$ http://www.lama.univ-savoie.fr/ lachaud/ImaGene/doc/html/index.html

${ }^{4}$ http://www. lama.univ-savoie.fr/ lachaud/ImaGene/doc/html/classImaGene_1_1C4CTangentialCover. html

${ }^{5}$ http://www . lama.univ-savoie.fr/ lachaud/ImaGene/doc/html/structImaGene_1_1C4CTangentialCover_ 1_1MaximalSegment.html

${ }^{6}$ http://www.lama.univ-savoie.fr/ lachaud/ImaGene/doc/html/classImaGene_1_1C4CIterator.html
} 


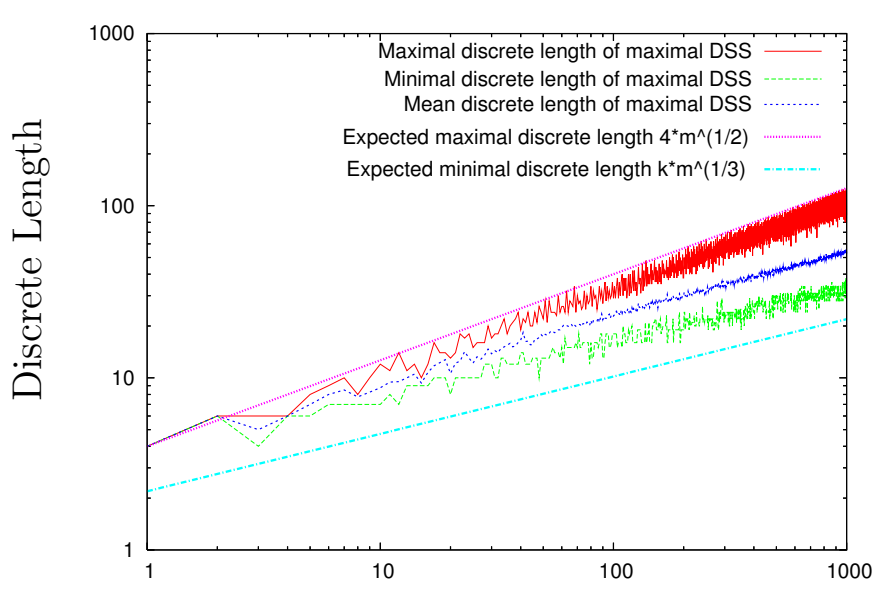

(a) Digitization size (h)

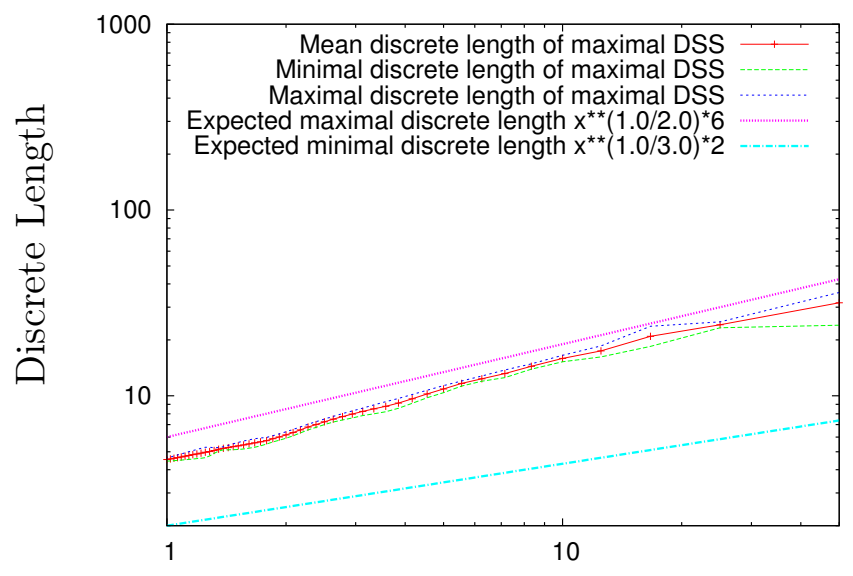

(b) Subsampling size

Figure 3: Experiments on measures of the length of maximal segments obtained from a flower shape for finer and finer grid size (a). Experiments on measure of the length of maximal segments obtained from a flower shape for different subsamplings (b).

\subsection{Meaningful Scale Detection from the Previous Property}

To exploit the previous property, we introduce the concept of multiscale profile defined for a contour point. A multiscale profile $\mathcal{P}_{n}(P)$ is defined as the sequence of samples $\left(\log (i), \log \left(\bar{L}^{h_{i}}\right)_{i=1 . . n}\right)$. The construction of such a multiscale profile is illustrated in figure 4 (a) with the contour sampled with grid size equal to 8 (represented in light blue).

A meaningful scale of a sequence $\left(S_{i}, T_{i}\right)_{1 \leq i \leq n}$ is defined as a pair $\left(i_{1}, i_{2}\right)$ such that:

$$
\forall i, i_{1} \leq i<i_{2}, \frac{T_{i+1}-T_{i}}{S_{i+1}-S_{i}} \leq t_{m}
$$

and the preceding property is not true for $i_{1}-1$ and $i_{2}$. The parameter $t_{m}$ is related to the previous theorem and this value has few influence towards the detection quality (see annex in the paper by Kerautret et al. [6] or section 5.2). The default value giving slightly better result was set to $t_{m}=0$. Note that from this definition the minimal length of the interval $\left(i_{1}, i_{2}\right)$ is one. Increasing this minimal value does not really improve the meaningful scale detection as you can see in section 5.2.

The noise level is defined as the first index for which the multiscale profile is going to decrease (i.e.) $i_{1}$. Figure 4 (c) illustrates the multiscale profile obtained on a point of a noisy contour part. The curve is first going the increase and then recover a normal behavior from the scale $i_{1}$. The noise level of this point is thus equal to $i_{1}$.

\section{Algorithm}

The algorithm for meaningful scale detection is composed of three main steps:

1. Subsampling the digital source contour to obtain the multiscale representation.

2. Tangential cover computation on all digital subsampled contours.

3. Computation of the multiscale profile and deduction of the noise level. 


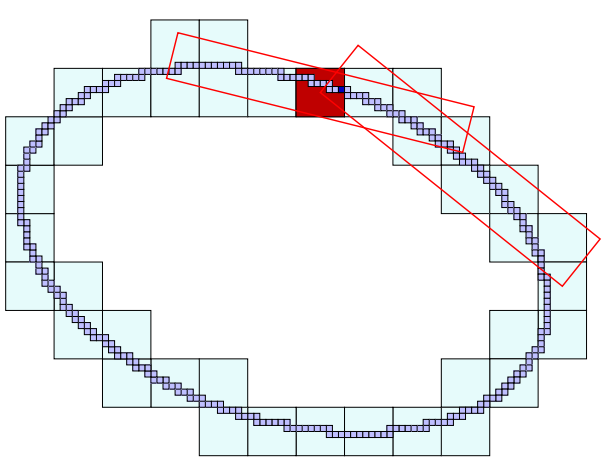

(a)

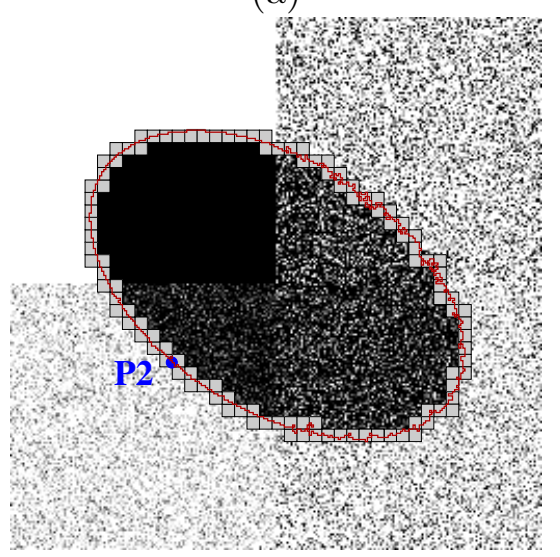

(c)

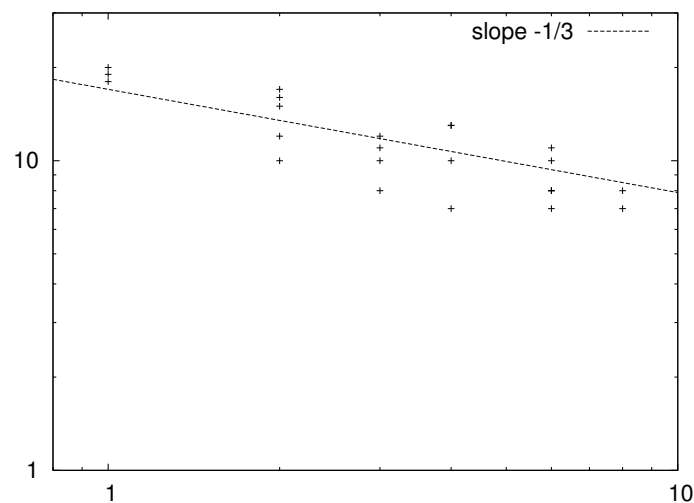

(b)

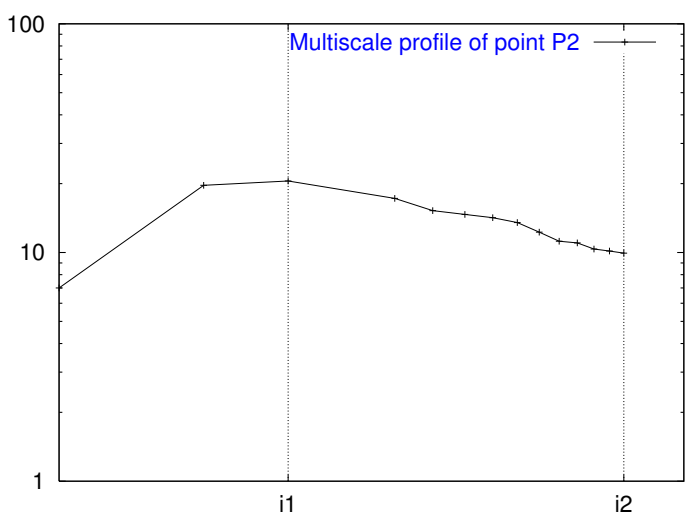

(d)

Figure 4: Images (a,b), construction of a multiscale profile with the set of maximal segments covering the red point for the grid size h equals to 8 . Images $(\mathrm{c}, \mathrm{d})$, illustration of a multiscale profile defined on a noisy contour point $\mathrm{P} 2$. The resulting meaningful scale is $\left(i_{1}, i_{2}\right)$.

\subsection{Subsampling the Discrete Source Contour}

Different methods are possible for the subsampling step. A common approach is to apply the subsampling by using the binary image of the contour and by covering it with a tilling of squares of size $i \times i$. Such an approach is simple but also presents the first inconvenient to not provide directly the correspondence between the digital contour of $\mathrm{O}$ and the digital contour of the subsampling of $\mathrm{O}$ (such a correspondence is illustrated in figure 5 (b)). A second inconvenient comes from the topological point of view since a simply connected shape can be transformed into a shape containing holes or vice versa.

We choose another approach by defining a contour based subsampling method. Given a digital 4connected contour $C$ that is the boundary of the set of pixels $O$ in the cellular model, the subsampling is performed in four steps:

- step 1: the interpixel contour $C$ is shifted toward the inside so that it defines the 4-connected inner border of $O$. This 4-connected contour of pixels is denoted by $C^{\prime}$. It is not necessarily simple and may contain some back-and-forth paths that are oriented toward the exterior of $O$ (outer spikes).

- step 2: the pixel contour $C^{\prime}$ is subsampled as the pixel contour $C$, composed of the sequence of points $\left(X_{j}, Y_{j}\right)=\left(\left(x_{j}-x_{0}\right) \div i,\left(y_{j}-y_{0}\right) \div i\right)$, where $C^{\prime}$ is the sequence of points $\left(x_{j}, y_{j}\right)$, $\left(x_{0}, y_{0}\right)$ represents the origin of the large pixels in $\mathbb{Z}^{2}$ and $\div$ is the integer division defined by 


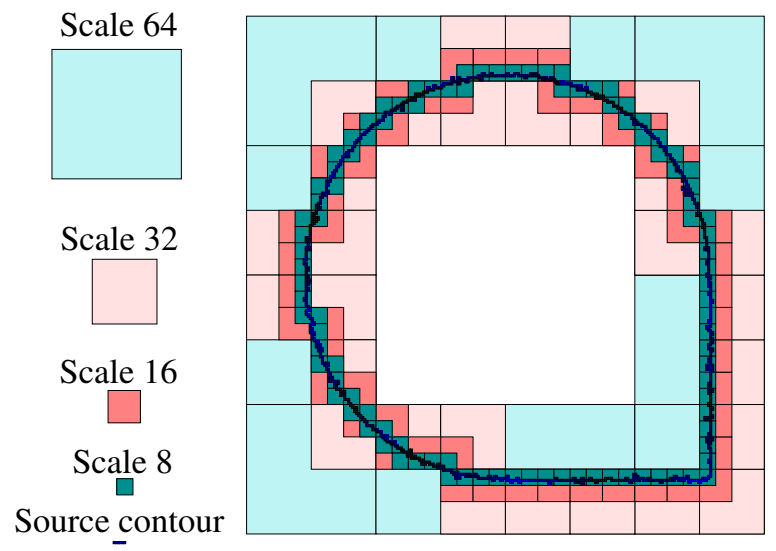

(a)

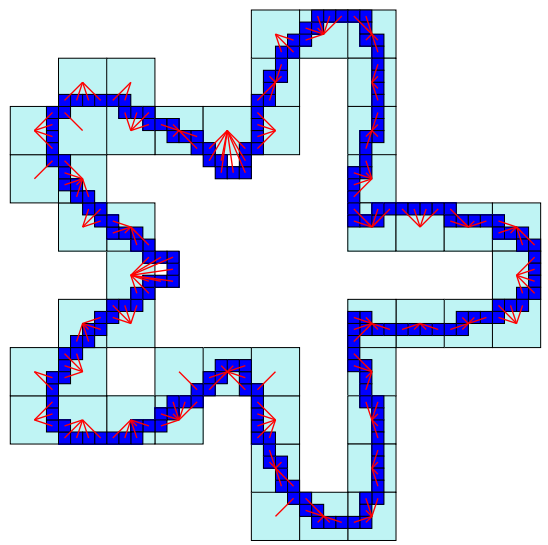

(b)

Figure 5: Image (a) illustrates a digital contour subsampling for $\mathrm{i}=8,16,32,64$ and image (b) gives an illustration of the function (represented by red lines) associating each pixel $\mathrm{P}$ of $\mathrm{C}$ to a point of the subsampled contour.

truncation.

- step 3: Consecutive identical pixels of $C^{\prime \prime}$ are merged to get a pixel contour $C^{\prime \prime \prime}$.

- step 4: Outer and inner spikes of $C^{\prime \prime \prime}$ are removed (by several linear scans). The obtained contour is shifted toward the outside so that it defines a 4-connected interpixel contour, that is, the boundary of some digital shape.

By following these main steps and from a given maximal scale $\mathrm{N}$, we are able to give as output the set $\mathcal{D}$ of the subsampled digital contours of $C$ (denoted as $\left.\phi_{i}^{x_{0}, y_{0}}\right)$. The function $f_{i}^{\left(x_{0}, y_{0}\right)}$ associating each contour point of $C^{\prime \prime \prime}$ should also be updated during steps 2, 3 and 4 .

Algorithm 1 summarizes the subsampling process which can be obtained with the object MultiscaleFreemanChain ${ }^{7}$ of the ImaGene framework.

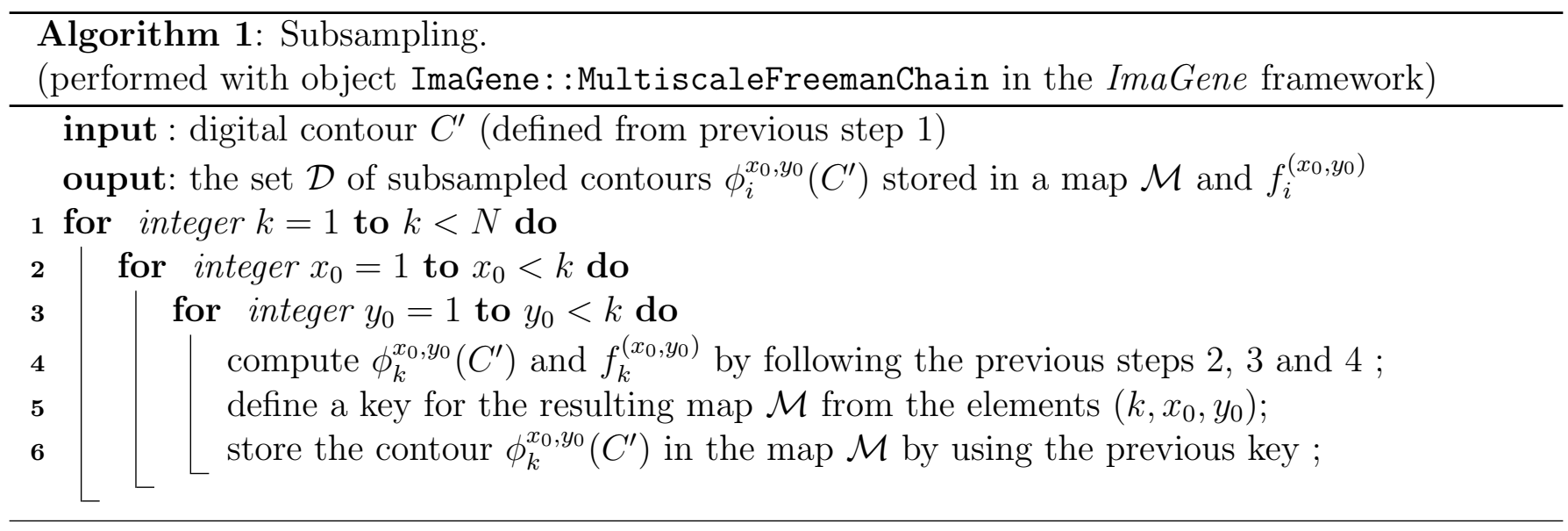

\subsection{Tangential Cover Computation and Length Statistic}

The set of all the maximal segments of a digital contour can be computed in linear time according the contour size [8]. Such an approach recognizes maximal segments by adding and removing points

\footnotetext{
${ }^{7}$ http://www. lama.univ-savoie.fr/ lachaud/ImaGene/doc/html/classImaGene_1_ 1MultiscaleFreemanChain.html
} 
from an initial one. The tangential cover should also be computed on all the subsampled contours.

Once all the maximal segments are computed for a given scale, the statistics of the maximal segment length are stored for each original contour point. The source contour point can be easily recovered by the function $f_{i}^{\left(x_{0}, y_{0}\right)}$.

The algorithm is summarized in algorithm 2. The implementation of this algorithm is included in ImaGene framework, in the class MultiscaleProfile from the directory ImaGene/helper.

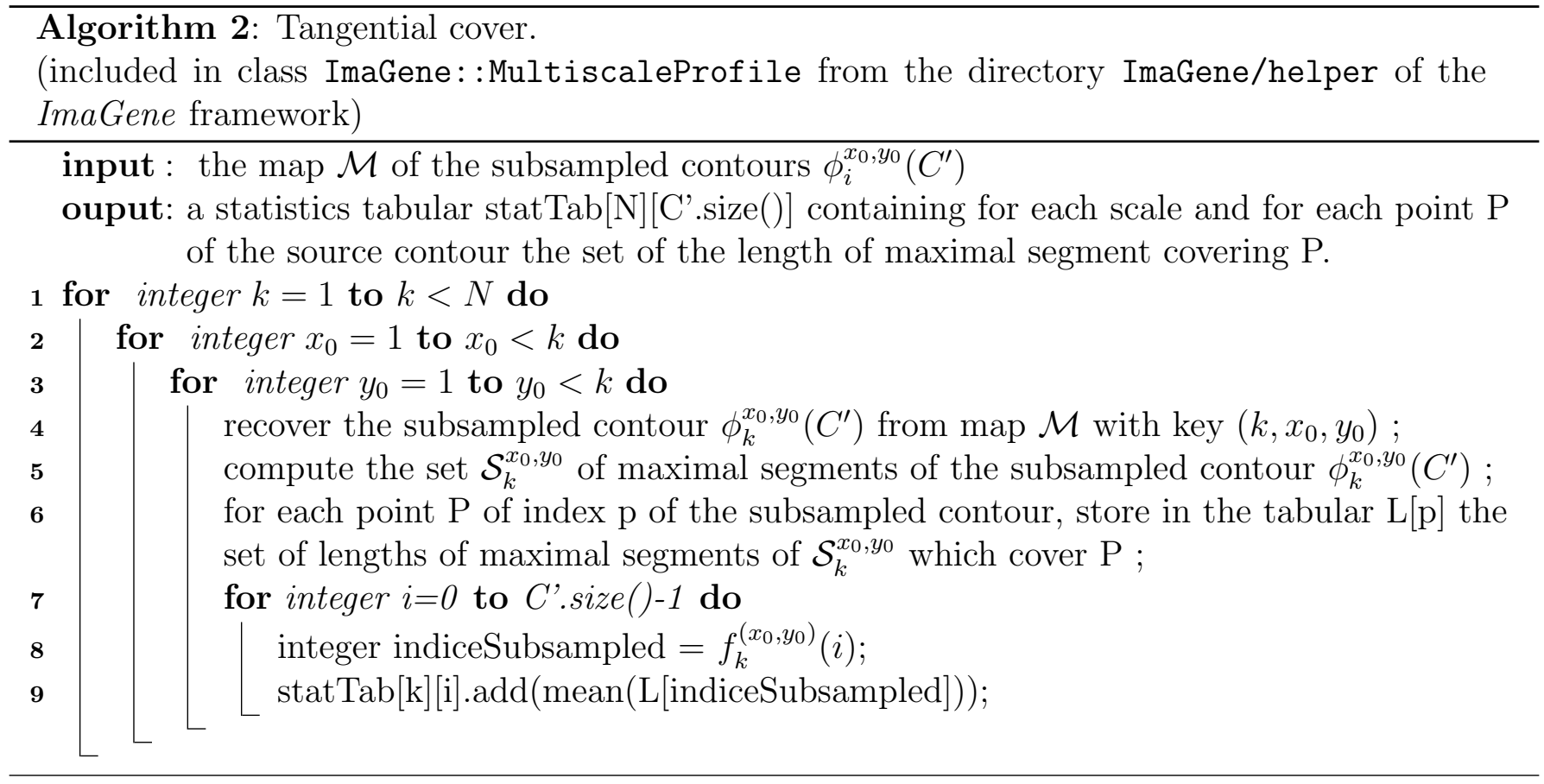

\subsection{Computation of the Multiscale Profile and Deduction of the Noise Level}

Once all the length statistics have been computed for each point of the source contour, we are able to determine a multiscale profile using algorithm 3.

The noise level can then be deduced from each multiscale profile (algorithm 4).

From this last algorithm, we are now able to give for each point of the discrete contour a noise level related to the meaningful scale detection.

\subsection{Complexity of the Global Algorithm}

The complexity of the global algorithm depends on the algorithm used to extract the set of maximal segments. Such extraction is implemented in linear time [3]. The tangential cover is computed for each scale $K$ which implies $K^{2}$ contour shifts. The global complexity is then $O\left(n K^{3}\right)$ with $n$ the number of contour points and $K$ the maximal scale. Note that this complexity could be improved by using a multiscale recognition of the tangential cover [12]. 

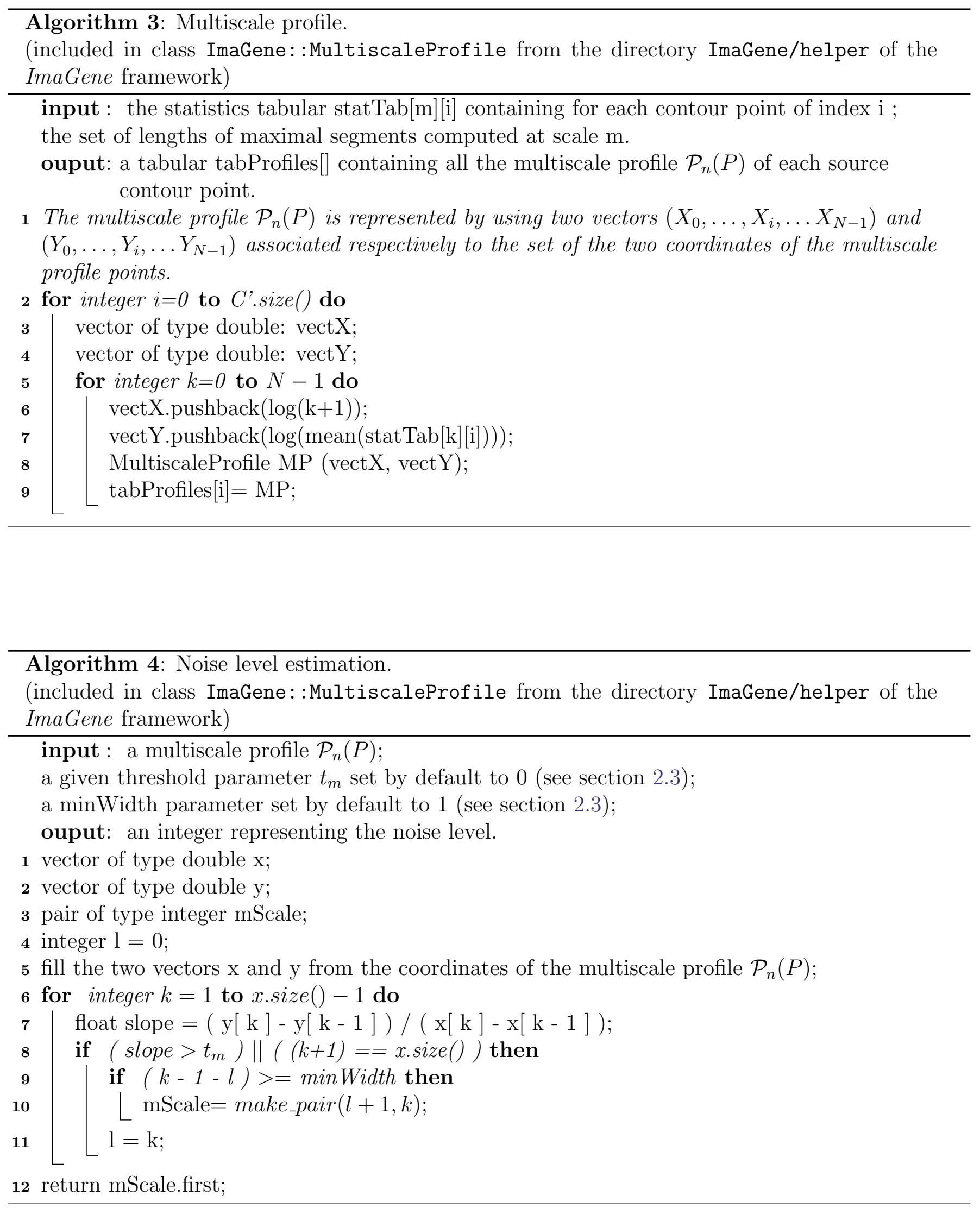


\section{Implementation}

\subsection{Source code}

The $\mathrm{C}++$ source code is provided in the ImaGene library ${ }^{8}$. A lighter version of this library has been constructed to avoid some dependencies with the boost and gmp libraries. The source code is available at the IPOL web page of this article ${ }^{9}$.

The installation uses cmake and was tested on Linux and Mac OSX. You just have to follow the README and INSTALL files.

\subsection{Input Format and Executable Test}

Input format. The input contour of the multiscale analysis should be represented in a simple Freeman chain format. Such a representation is defined as follows: XO YO COC1C2C2 ...CN. The two first integers $\mathrm{X} 0$ and $\mathrm{Y} 0$ represent the coordinates of the initial contour point. The other numbers $\mathrm{Ci}$ represent the directions of the move to go from the current point to the next one. The code convention is the following:

- code 0: move left $(x+1)$

- code 1: move up $(y+1)$

- code 2: move left (x-1)

- code 3: move down $(\mathrm{y}-1)$

Such contour examples are given in the directory meaningfulscaleDemo/demoIPOL/Contours with the ".fc" file extension. The file meaningfulScaleEstim located in the directory meaningfulscaleDemo/demoIPOL can be used to detect noise from the meaningful scales.

Noise detection test program. To perform the noise detection you can use the following command lines:

cd meaningfulscaleDemo/build/demoIPOL

./meaningfulScaleEstim < ../. ./demoIPOL/Contours/ellipseBruit.fc $-\hookleftarrow$ drawXFIGNoiselevel -enteteXFIG -drawContourSRC $41>$ tmp.fig

The previous command generates an xfig file which can be transformed with ${ }^{10}$ :

fig2dev -L eps tmp.fig tmp.eps

You should obtain the results presented in figure 6 .

\subsection{Including Meaningful Scale Detection in Other Programs}

To include the meaningful scale detection in a $\mathrm{C}++$ program with the ImaGene library, you need first to include the following header files:

\footnotetext{
${ }^{8}$ http://gforge.liris.cnrs.fr/projects/imagene

${ }^{9}$ https://doi.org/10.5201/ipol.2014.75

${ }^{10}$ the $\mathrm{fig} 2 \mathrm{dev}$ command can be found in the transfig package
} 


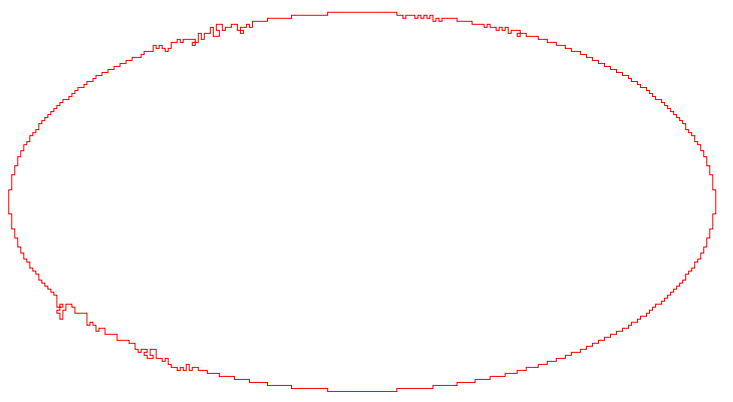

(a)

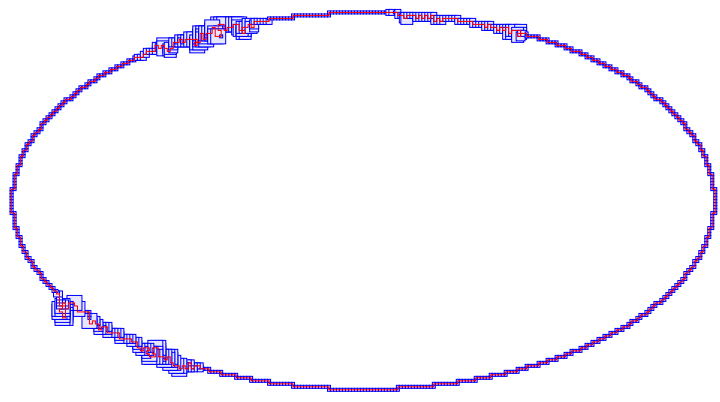

(b)

Figure 6: (a) Source contour of the Freeman chain ellipseBruit.fc located in the directory demoIPOL/Contours and (b) Meaningful scale results represented with blue boxes of size equal to the noise level and centered on each contour point.

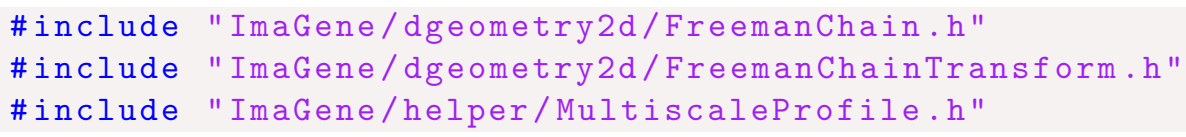

and then follow these steps:

- Step 1: read the Freeman chain from the standard input. And then read the Freeman chain from input:

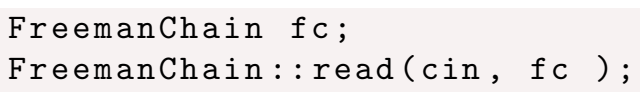

- Step 2: construction of the multiscale profile.

This step needs the setting of the parameter associated to the maximal scale for which the shape is analyzed. Since we are just searching for the first meaningful scale, the maximal scale is not really important and depends only of what maximal amount of noise you have to detect (for instance 10 looks sufficient in most cases). The variable nbIterationSpikeDetection is used to avoid degenerated contours and a value of 5 appears sufficient for all experiments.

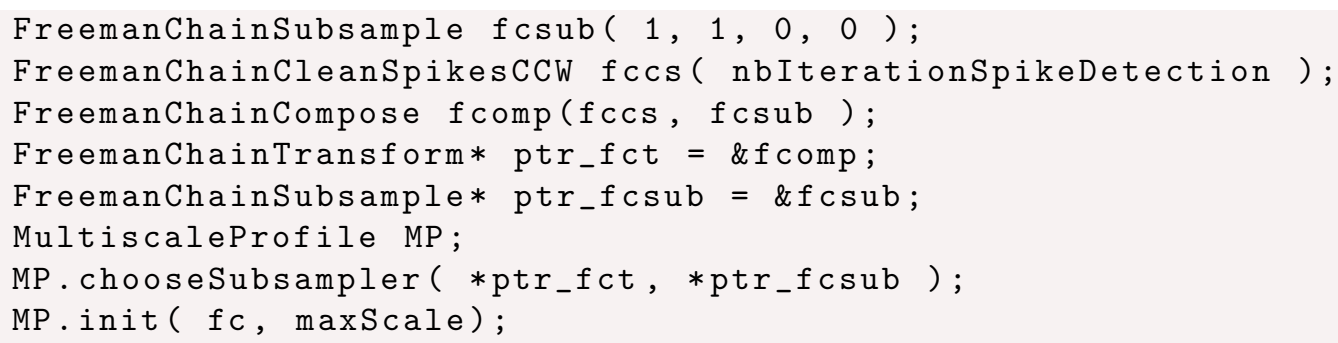

- Step 3: estimate and display noise levels.

The minSize and maxSlope values should be set to the default value, respectively to 1 and 0 .

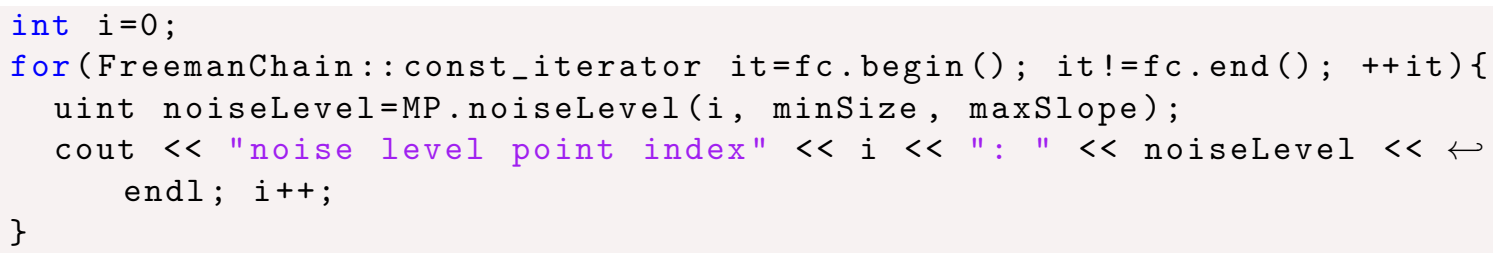


This simple minimal example is given in the meaningfulscaleDemo archive in the file minimalCodeNoiseDetection.cxx of the directory meaningfulscaleDemo/demoIPOL/.

\section{$5 \quad$ Examples and Experiments}

\subsection{Examples on Various Noisy Shapes}

We present experiments obtained with various noisy shapes by using the default intern parameters $t_{m}=0.0$, max $_{s}$ scale $=15$ and minimal meaningful scale size min_size=1. Figures 7 and 8 show the results obtained on digital contours extracted from binary and grayscale images. The contours extraction was performed from the multidimensional algorithm based on boundary extraction and contour tracking [2] which is available from the IPOL site ${ }^{11}$. This extraction is also implemented in the $I m$ aGene framework and given in the source demo in: meaningfulscaleDemo/bin/pgm2freeman.cxx.

\subsection{Stability Measure from Internal Parameters}

Even if the meaningful scale detection can be considered as parameter free, it contains some internal parameters which have only small influence on the detection quality. To demonstrate this stability, several experiments were performed on the shapes of figure 9 (a,c) for which the default meaningful scale detection is given on images $(b, d)$. First we experiment the influence of the change of each particular parameter and then the change of all parameters together.

Experiments with the change of the threshold of the minimal slope $\left(t_{m}\right)$. As introduced in section 2.3 a threshold $t_{m}$ was defined to discriminate smooth and noisy contour parts. By default, the value was set to 0 and as it can be seen in figure 10 (two first upper rows), modification of this particular parameter does not really change the global quality of the noise detection.

Experiments by adding a minimal length for the meaningful scale interval (msMinLength). In the definition of the meaningful scale of section 2.3, a minimal size of one was introduced to define the interval $\left(i_{1}, i_{2}\right)$. Figure 10 (two last lower rows) shows the results obtained by changing this definition. As illustrated, the change of the initial definition does not really improve the meaningful scale detection and with too large values of the scale interval some corners tend to be detected as noise.

These experiments with the change of the $t_{m}$ and msMinLength can be reproduced by the following command line from the meaningfulscaleDemo/build directory:

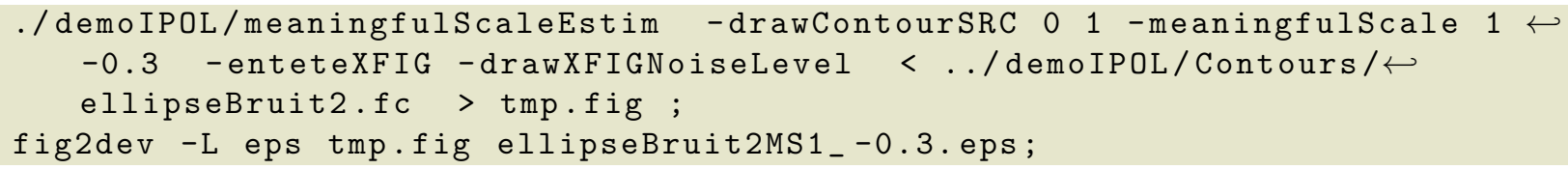

where the option -meaningfulScale permits to set the parameters associated to minimal length for the meaningful scale definition and the threshold $t_{m}$.

Experiments with the change of the analysis maximal scale (maxScale). A last internal parameter is the maximal scale for which the shape is analyzed. Since the noise level is defined as the first scale of the meaningful scale interval, a change of this value has small influence. The experiments

\footnotetext{
${ }^{11}$ http://www.ipol.im
} 

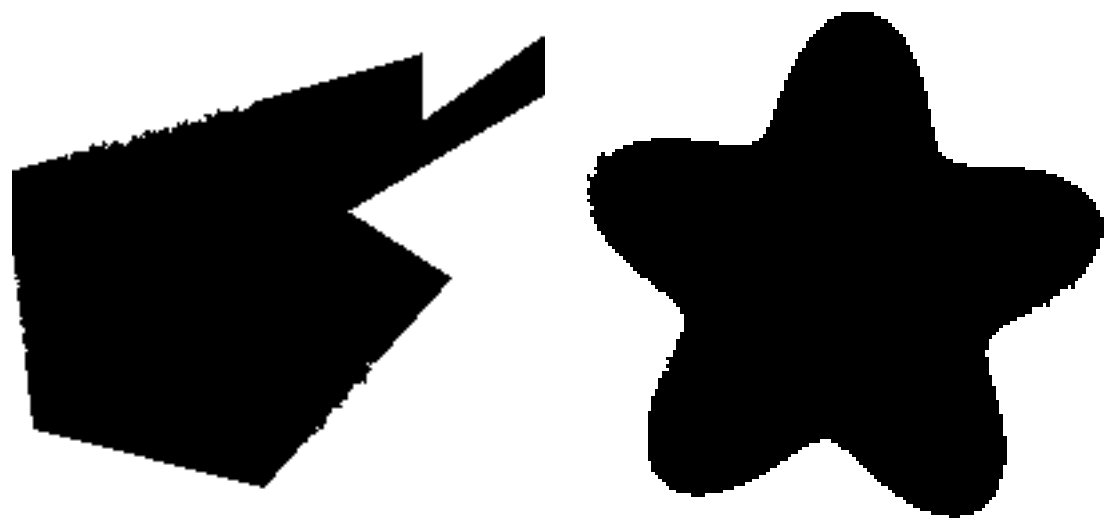

source contour 1
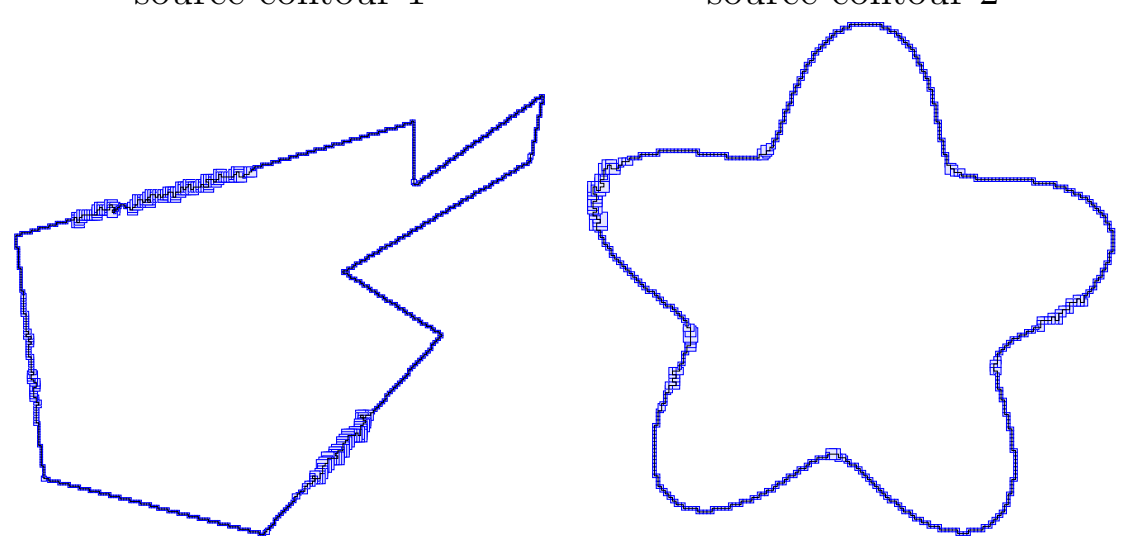

meaningful scale

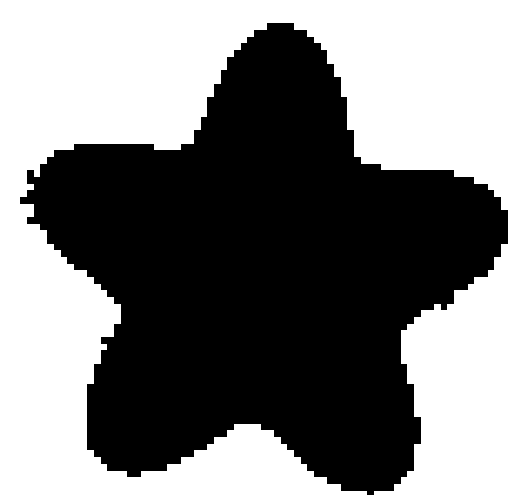

source contour 3

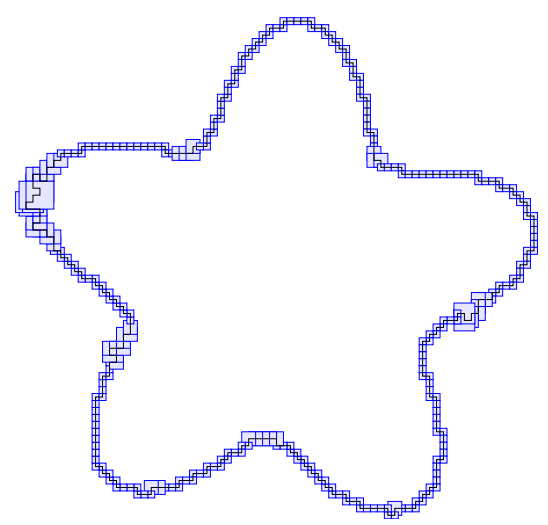

meaningful scale
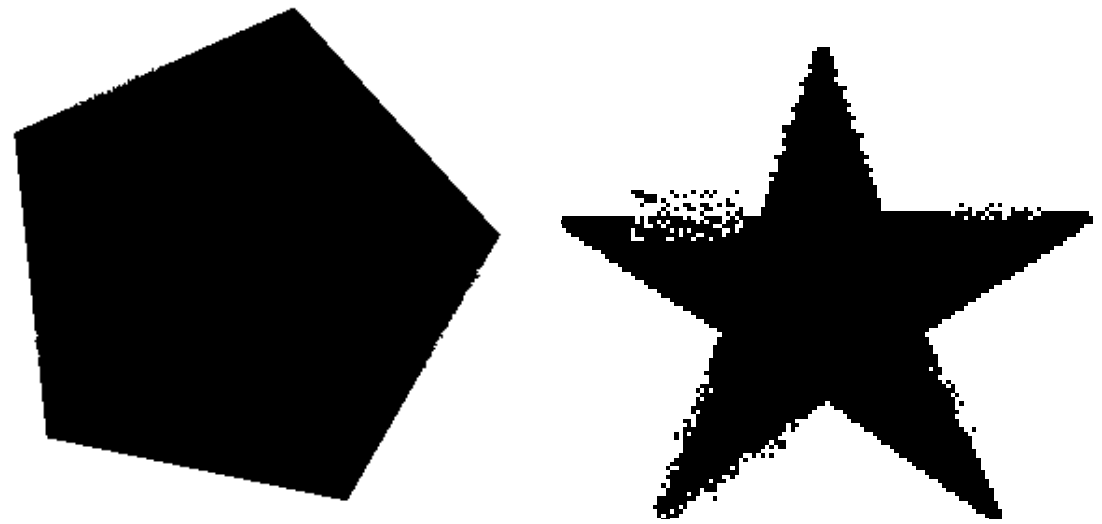

source contour 5

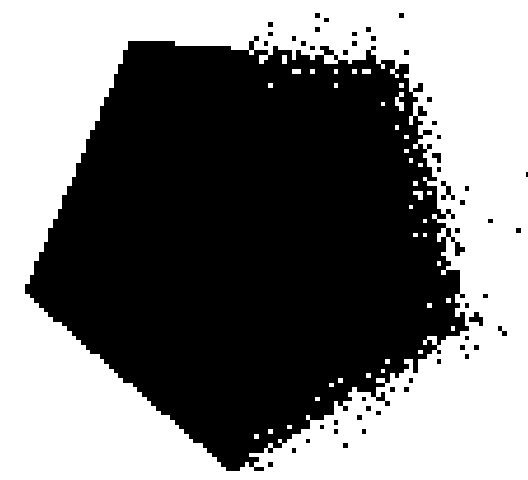

source contour 6

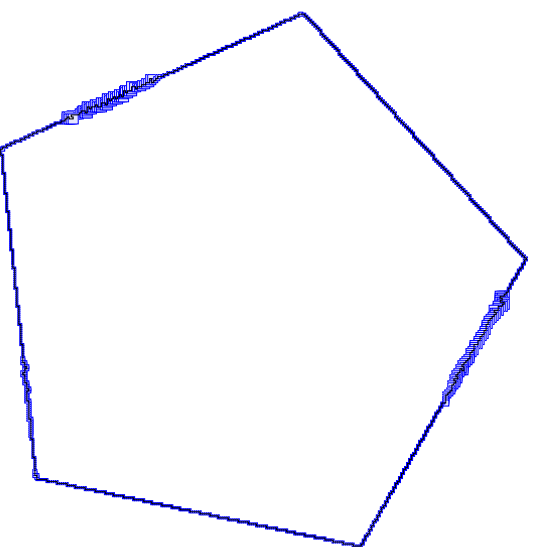

meaningful scale

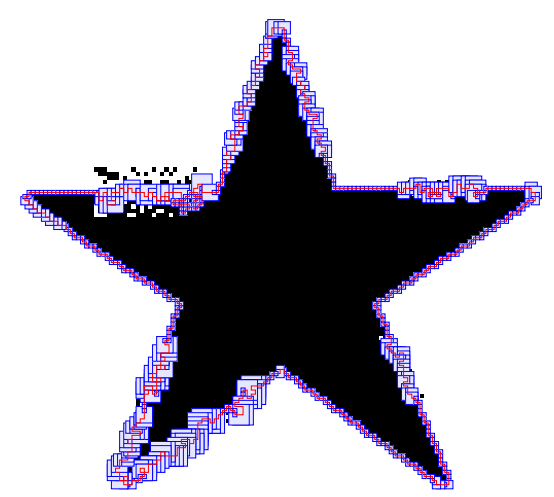

meaningful scale

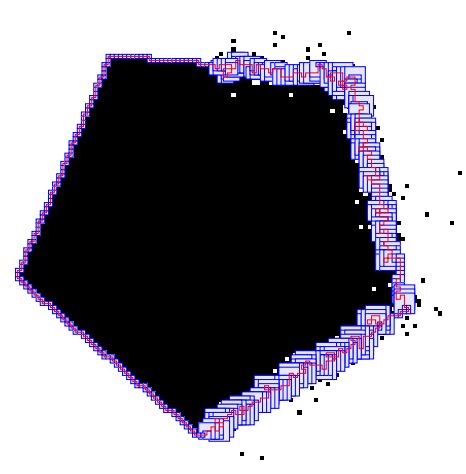

meaningful scale

Figure 7: Illustration of the meaningful scale detection on some noisy shapes. The meaningful scales are represented with blue boxes of size equal to the noise level and centered on each contour point. 


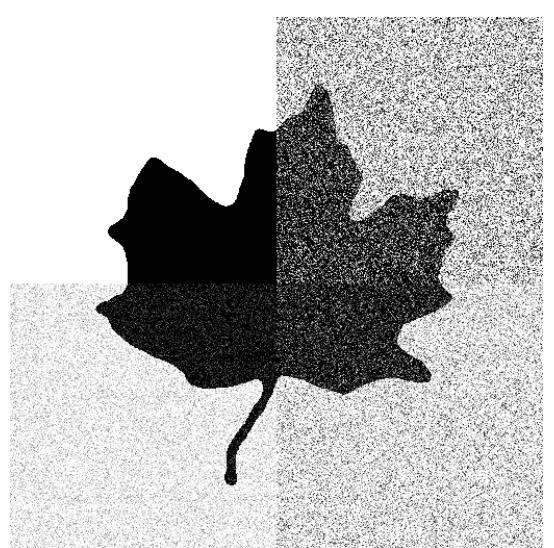

source contour 7

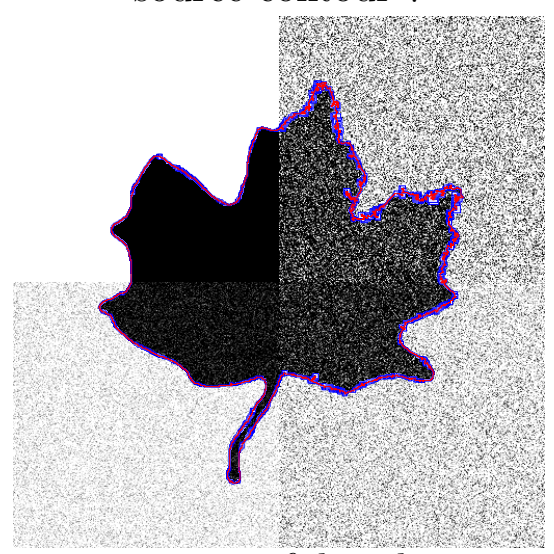

meaningful scale

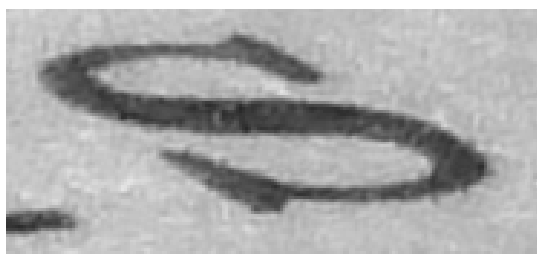

source contour 8

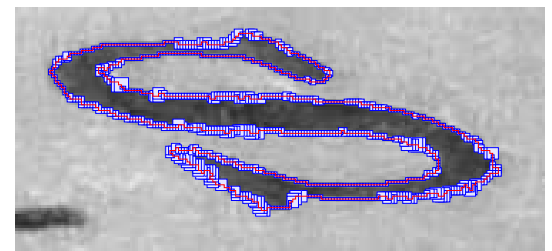

meaningful scale

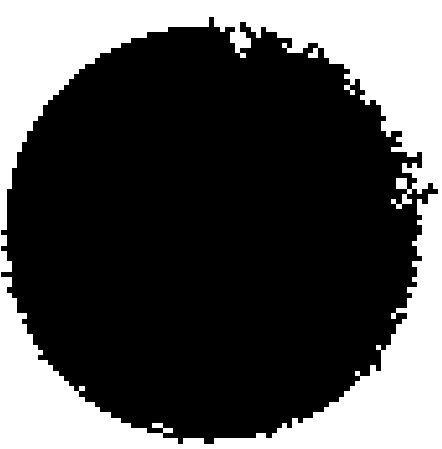

source contour 9

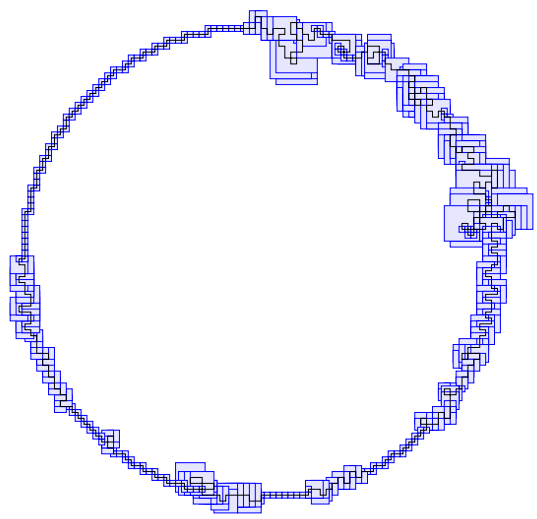

meaningful scale

Figure 8: Illustration of the meaningful scale detection on some noisy shapes. The meaningful scales are represented with blue boxes of size equal to the noise level and centered on each contour point.

presented in figure 11 (two first upper rows) confirm this fact. Note that the areas displayed in green correspond to the points for which no meaningful scale was found below the maximal scale. In this particular case a box is displayed with the given maximal scale.

These experiments can be reproduced by the following command lines from the meaningfulscaleDemo/build directory:

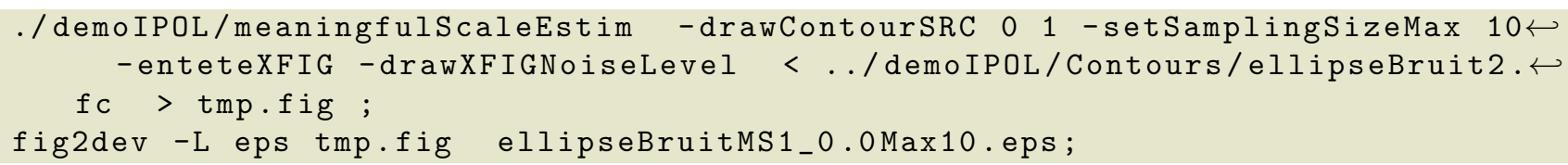

where the analysis maximal scale (parameter maxScale) is set with option -setSamplingSizeMax.

Experiments with the change of all the parameters together. To apply a last test on the independence towards the internal parameters, we apply the meaningful scale detection with various changes on all the three parameters. The lower part of figure 11 shows globally good results even if with the case of parameters (msMinLength $=4, t_{m}=-0.1$, maxScale $=20$ ) one area between two corners is detected as noise. 


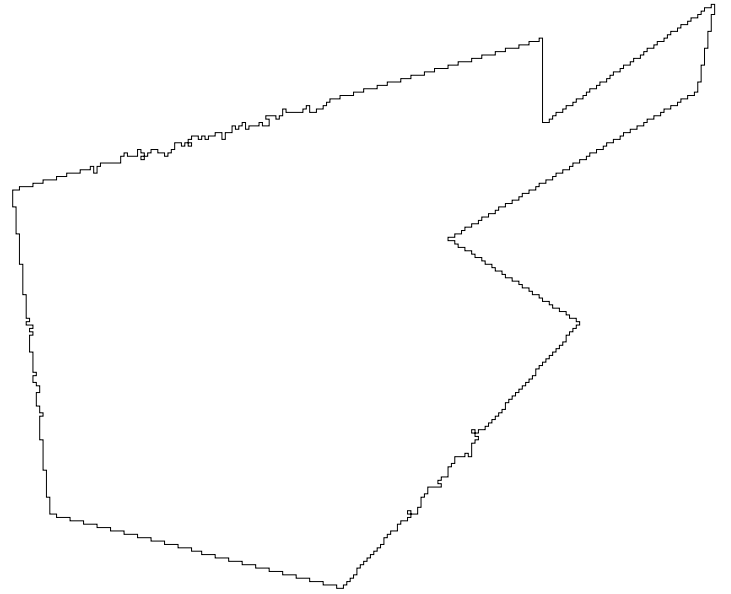

(a) source

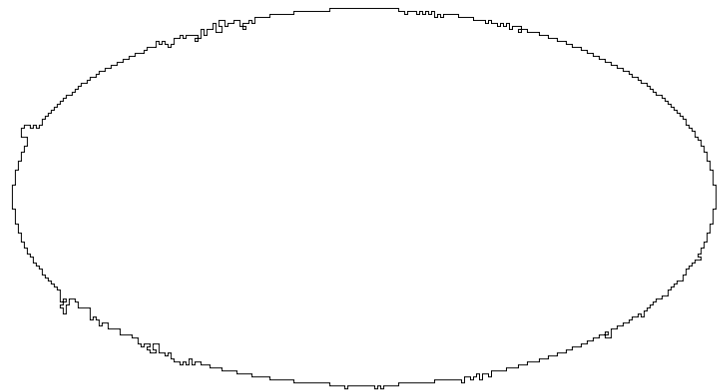

(c) source

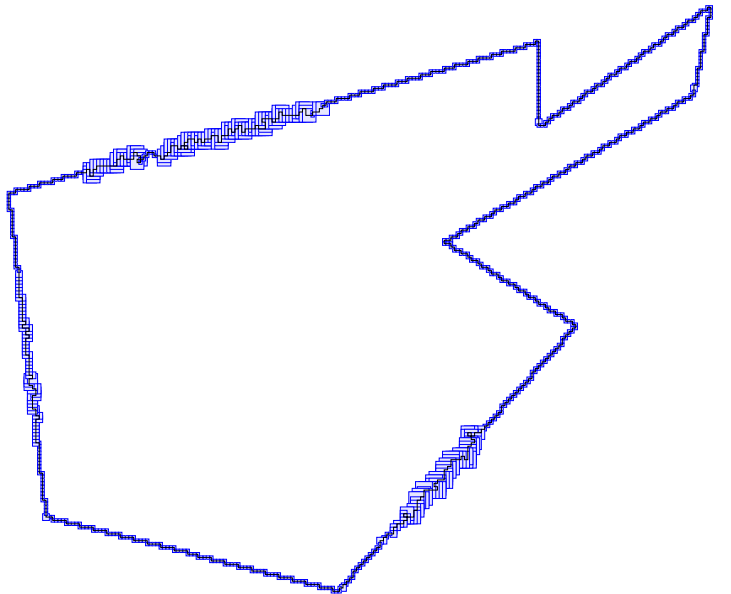

(b) result (default parameters)

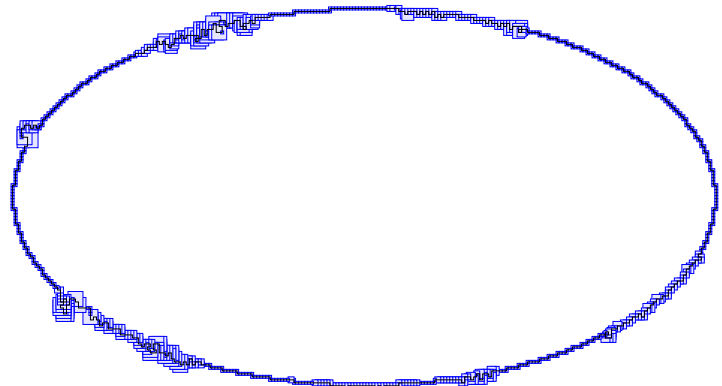

(d) result (default parameters)

Figure 9: Noise estimation with the default parameters on the polygon (a,b) and ellipse shapes (c,d). 


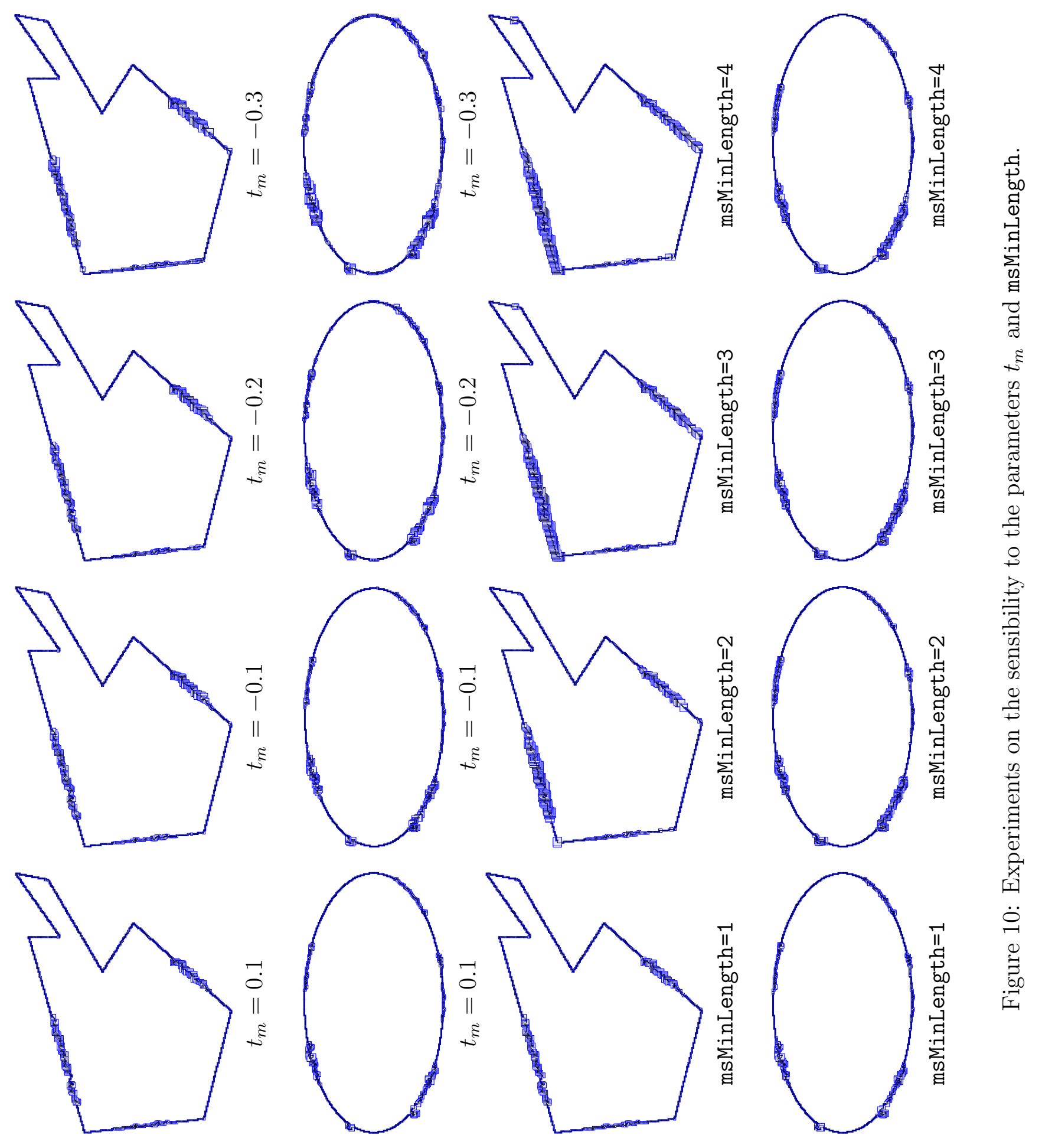




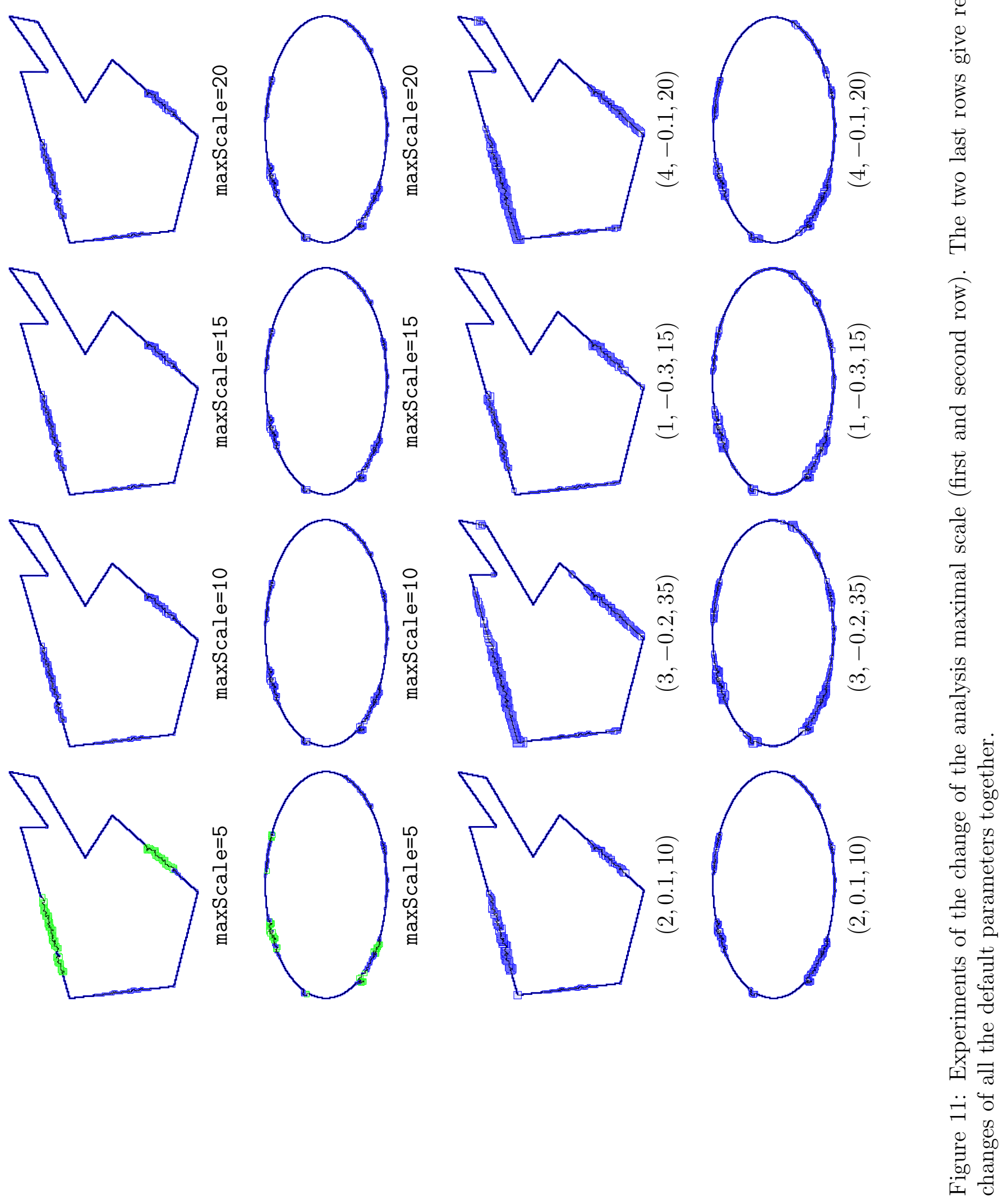




\section{Image Credits}

All images and contours created by the authors except:

which is a standard test contour and

which is a noisy version of a standard test image.

\section{References}

[1] P. Bhowmick And B. B. Bhattacharya, Fast polygonal approximation of digital curves using relaxed straightness properties, IEEE Transactions on Pattern Analysis and Machine Intelligence, 29 (2007), pp. 1590-1602. http://dx.doi.org/10.1109/TPAMI.2007.1082.

[2] D. Coeurjolly, B. Kerautret, and J.-O. Lachaud, Extraction of connected region boundary in multidimensional images, Image Processing On Line, (2014). http://dx.doi.org/10. 5201/ipol.2014.74.

[3] F. Feschet And L. Tougne, Optimal time computation of the tangent of a discrete curve: Application to the curvature, in Discrete Geometry for Computer Imagery, Gilles Bertrand, Michel Couprie, and Laurent Perroton, eds., vol. 1568 of Lecture Notes in Computer Science, Springer Berlin Heidelberg, 1999, pp. 31-40. http://dx.doi.org/10.1007/3-540-49126-0_3.

[4] B. Kerautret And J.-O. Lachaud, Curvature estimation along noisy digital contours by approximate global optimization, Pattern Recognition, 42 (2009), pp. 2265-2278. http://dx. doi.org/10.1016/j.patcog.2008.11.013.

[5] _ Multi-scale analysis of discrete contours for unsupervised noise detection, in Proceedings of the 13th international Workshop on Combinatorial Image Analysis (IWCIA), P. Wiederhold and R.P. Barneva, eds., vol. 5852 of LNCS, Springer, November 2009, pp. 187-200. http: //dx.doi.org/10.1007/978-3-642-10210-3_15.

[6] _ Meaningful scales detection along digital contours for unsupervised local noise estimation, IEEE Transactions on Pattern Analysis and Machine Intelligence, 34 (2012), pp. 2379-2392. http://dx.doi.org/10.1109/TPAMI.2012.38.

[7] J.-O. LACHAUD, Espaces non-euclidiens et analyse d'image : modèles déformables riemanniens et discrets, topologie et géométrie discrète, habilitation à diriger des recherches, Université Bordeaux 1, Talence, France, 2006. (in french).

[8] J.-O. Lachaud, A. Vialard, and F. De Vieilleville, Fast, accurate and convergent tangent estimation on digital contours, Image and Vision Computing, 25 (2007), pp. 1572-1587. http://dx.doi.org/10.1016/j.imavis.2006.06.019.

[9] H. LiU, L. LATECKI, AND W. LiU, A unified curvature definition for regular, polygonal, and digital planar curves, International Journal of Computer Vision, 80 (2008), pp. 104-124. http: //dx.doi.org/10.1007/s11263-008-0131-y.

[10] T.P. Nguyen and I. Debled-Rennesson, Curvature estimation in noisy curves, in Computer Analysis of Images and Patterns, WalterG. Kropatsch, Martin Kampel, and Allan Hanbury, eds., vol. 4673 of Lecture Notes in Computer Science, Springer Berlin Heidelberg, 2007, pp. 474-481. http://dx.doi.org/10.1007/978-3-540-74272-2_59. 
[11] T.P. Nguyen and I. Debled-Rennesson, A discrete geometry approach for dominant point detection, Pattern Recognition, 44 (2011), pp. 32-44. http://dx. doi.org/10.1016/j.patcog. 2010.06 .022 .

[12] M. SAID AND J.-O. Lachaud, Computing the characteristics of a subsegment of a digital straight line in logarithmic time, in Discrete Geometry for Computer Imagery, Isabelle DebledRennesson, Eric Domenjoud, Bertrand Kerautret, and Philippe Even, eds., vol. 6607 of Lecture Notes in Computer Science, Springer Berlin Heidelberg, 2011, pp. 320-332. http://dx.doi. org/10.1007/978-3-642-19867-0_27. 